\title{
Perioperative FOLFOX4 plus bevacizumab for initially unresectable advanced colorectal cancer (NAVIGATE-CRC-OI)
}

This article was published in the following Dove Press journal:

OncoTargets and Therapy

18 May 2015

Number of times this article has been viewed

\author{
Mitsukuni Suenaga' \\ Yoshiya Fujimoto² \\ Satoshi Matsusaka' \\ Eiji Shinozaki' \\ Takashi Akiyoshi ${ }^{2}$ \\ Satoshi Nagayama ${ }^{2}$ \\ Yosuke Fukunaga ${ }^{2}$ \\ Masatoshi Oya ${ }^{2}$ \\ Masashi Ueno ${ }^{2}$ \\ Nobuyuki Mizunuma' \\ Toshiharu Yamaguchi
}

'Department of Gastroenterology, ${ }^{2}$ Department of Gastroenterological Surgery, Cancer Institute Hospital, Japanese Foundation for Cancer Research, Tokyo, Japan
Correspondence: Mitsukuni Suenaga Department of Gastroenterology, Cancer Institute Hospital, Japanese Foundation for Cancer Research, 3-8-3I Ariake, Koto-ku, Tokyo 135-8550, Japan

$\mathrm{Tel}+8 \mathrm{I} 335200$ III

$\mathrm{Fax}+8 \mathrm{I} 335700343$

Emailm.suenaga@jfcr.or.jp
Background: Perioperative chemotherapy combined with surgery for liver metastases is considered an active strategy in metastatic colorectal cancer (CRC). However, its impact on initially unresectable, previously untreated advanced CRC, regardless of concurrent metastases, remains to be clarified.

Methods: A Phase II study was conducted to evaluate the safety and efficacy of perioperative FOLFOX4 plus bevacizumab for initially unresectable advanced CRC. Patients with previously untreated advanced colon or rectal cancer initially diagnosed as unresectable advanced CRC (TNM stage IIIb, IIIc, or IV) but potentially resectable after neoadjuvant chemotherapy (NAC) were studied. Preoperatively, patients received six cycles of NAC (five cycles of neoadjuvant FOLFOX4 plus bevacizumab followed by one cycle of FOLFOX4 alone). The interval between the last dose of bevacizumab and surgery was at least 5 weeks. Six cycles of adjuvant FOLFOX4 plus bevacizumab were given after surgery. The completion rate of NAC and feasibility of curative surgery were the primary endpoints.

Results: An interim analysis was performed at the end of NAC in the 12th patient to assess the completion rate of NAC. The median follow-up time was 56 months. The characteristics of the patients were as follows: sex, eight males and four females; tumor location, sigmoid colon in three, ascending colon in one, and rectum (above the peritoneal reflection) in eight; stage, III in eight and IV in four (liver or lymph nodes). All patients completed six cycles of NAC. There were no treatment-related severe adverse events or deaths. An objective response to NAC was achieved in nine patients (75\%), and no disease progression was observed. Eleven patients underwent curative tumor resection, including metastatic lesions. In December 2012, this Phase II study was terminated because of slow registration.

Conclusion: Perioperative FOLFOX4 plus bevacizumab is well tolerated and has a promising response rate leading to curative surgery, which offers a survival benefit in initially unresectable advanced CRC with concurrent metastatic lesions.

Keywords: bevacizumab, metastatic colorectal cancer, FOLFOX4, perioperative treatment

\section{Introduction}

Colorectal cancer is the leading cause of death in women and third leading cause of death in men in Japan. ${ }^{1}$ In the last decade, highly promising, new molecularly targeted agents have been introduced for the treatment of metastatic colorectal cancer (CRC). Four molecular targeted agents have been approved in Japan, ie, bevacizumab, cetuximab, panitumumab, and regorafenib. Moreover, a novel oral nucleotide antitumor agent (TAS-102) was recently approved only in Japan. ${ }^{2}$ Japanese patients with metastatic CRC can thus receive evidence-based treatment with a range of cytotoxic and molecular targeted agents under national health insurance. Bevacizumab is a 
recombinant, humanized monoclonal antibody against vascular endothelial growth factor. When used in combination with chemotherapy, bevacizumab has been shown to improve survival as both first-line and second-line treatment in patients with metastatic CRC. ${ }^{3-7}$ Thus, chemotherapy plus bevacizumab has been established to be the optimal standard regimen for metastatic $\mathrm{CRC}$.

Perioperative chemotherapy combined with surgery for liver metastases is regarded as an effective strategy in metastatic CRC. However, its impact on initially unresectable or potentially resectable, previously untreated advanced CRC regardless of the presence or absence of concurrent metastases remains to be clarified. ${ }^{8,9}$ Preoperative chemotherapy is thought to offer the following benefits: eradication of micrometastases or circulating tumor cells; reduction in surgical tumor shedding; maintenance of the intensity of chemotherapy; and evaluation of the safety of chemotherapy and chemosensitivity, contributing to assessing the need for postoperative chemotherapy or selection of the chemotherapy regimen. On the other hand, preoperative chemotherapy has the following potential disadvantages: a risk of overtreatment due to inaccurate radiological staging, leading to severe toxicity in low-risk patients; a risk of bowel obstruction caused by the primary tumor during preoperative therapy, resulting in emergency but not radical surgery; and an increased risk of perioperative complications.

After approval of bevacizumab in Japan in 2007 for the treatment of metastatic CRC, we conducted this Phase II study to investigate the safety and efficacy of perioperative chemotherapy plus bevacizumab in patients with initially unresectable advanced CRC.

\section{Patients and methods Study design}

This prospective Phase II study was performed at a single center in Japan. We evaluated the safety and efficacy of perioperative FOLFOX4 plus bevacizumab in patients with initially unresectable advanced CRC. The study was performed in accordance with the Declaration of Helsinki and the ethical guidelines for clinical studies. The institutional review board at the study site approved the protocol. This study has been registered in the UMIN Clinical Trials Registry as UMIN000005572.

\section{Patients}

Eligible patients had to have a histologically confirmed diagnosis of previously untreated advanced colon cancer arising in the rectosigmoid or the rectum above the peritoneal reflection, initially considered unresectable or marginally resectable
(TNM stage IIIb, IIIc, or IV, 6th Edition), indicating potentially resectable after neoadjuvant chemotherapy (NAC). Concerning IIIb or IIIc, unresectable was defined that the surgical margin of primary tumor or lymph node was considered to be positive for cancer. Radiological metastases to four or more regional lymph nodes (N2) or at least one main lymph node (N3) by computed tomography or magnetic resonance imaging according to the general rules for clinical and pathological studies on cancer of the colon, rectum and anus (Japanese Society for Cancer of the Colon and Rectum, 7th Edition) ${ }^{10}$ were also eligible. All patients also had to meet the following criteria: age $\geq 20$ years; Eastern Cooperative Oncology Group performance status 0 or 1 ; neutrophil count $\geq 1,500 / \mathrm{mm}^{3}$; platelet count $\geq 100,000 / \mathrm{mm}^{3}$; hemoglobin level $\geq 9.0 \mathrm{~g} / \mathrm{dL}$; total bilirubin $\leq 1.5$ times the institution's upper limit of normal (ULN); aspartate aminotransferase, alanine aminotransferase, and alkaline phosphatase $\leq 2.5$ times the ULN; serum creatinine $\leq 1.5$ times the ULN; and be able to provide signed informed consent.

Patients with any of the following conditions were excluded: clinically detectable ascites; brain tumors or brain metastases; major surgery, open biopsy, clinically significant traumatic injury within 4 weeks before enrollment or fineneedle aspiration biopsy within 1 week before enrollment; a bleeding diathesis or coagulopathy; active gastrointestinal ulcer; current or previous (within the last year) history of gastrointestinal perforation; international normalized ratio $\leq 1.5$ within 2 weeks before enrollment; non-healing bone fracture; urinary protein $\geq 1+$ within 2 weeks before enrollment; uncontrolled hypertension; clinically significant cardiovascular disease; long-term treatment with high-dose aspirin ( $\geq 325 \mathrm{mg} /$ day) or non-steroidal anti-inflammatory medications; severe peripheral neuropathy; or uncontrolled active infection.

\section{Chemotherapy}

The FOLFOX4 plus bevacizumab regimen was given every 2 weeks. On day 1 , oxaliplatin $85 \mathrm{mg} / \mathrm{m}^{2}$ was given as a 2-hour intravenous infusion, and bevacizumab $5 \mathrm{mg} / \mathrm{kg}$ was given as an intravenous infusion over the course of 30-90 minutes. On days 1 and 2, L-leucovorin $100 \mathrm{mg} / \mathrm{m}^{2}$ was given as a 2-hour intravenous infusion (concurrently with oxaliplatin), followed immediately by an intravenous bolus injection of 5-fluorouracil $400 \mathrm{mg} / \mathrm{m}^{2}$ and a 22-hour intravenous infusion of 5-fluorouracil $600 \mathrm{mg} / \mathrm{m}^{2}$. Patients received six cycles of NAC. The sixth cycle did not include bevacizumab. The interval between the last dose of bevacizumab and surgery had to be at least 5 weeks. After surgery, 
patients received six cycles of adjuvant FOLFOX4 plus bevacizumab.

The study treatment was delayed if either of the following criteria were not met on the scheduled day of administration or the previous day: neutrophil count $\geq 1,500 / \mathrm{mm}^{3}$ or platelet count $\geq 100,000 / \mathrm{mm}^{3}$. The oxaliplatin dose was reduced to $65 \mathrm{mg} / \mathrm{m}^{2}$ if grade 4 neutropenia or grade 3 or higher thrombocytopenia, nausea/vomiting, or diarrhea occurred. Oxaliplatin was withdrawn if severe neurotoxicity persistently occurred. As for the doses of 5-fluorouracil, the infusion dose was reduced to $500 \mathrm{mg} / \mathrm{m}^{2}$ or $400 \mathrm{mg} / \mathrm{m}^{2}$ and the bolus dose to $300 \mathrm{mg} / \mathrm{m}^{2}$ or $200 \mathrm{mg} / \mathrm{m}^{2}$ in patients who had grade 4 neutropenia or grade 3 or higher thrombocytopenia, nausea/ vomiting, diarrhea, stomatitis, or hand-foot syndrome. No dose modifications were allowed for bevacizumab. The study was terminated if bevacizumab was permanently discontinued because of grade 3 or higher thrombus formation, any grade hemoptysis or gastrointestinal perforation, refractory grade 2 or higher proteinuria or uncontrolled hypertension, or refractory grade 2 or grade 3 or higher bleeding, or reversible posterior leukoencephalopathy syndrome. The study was also terminated in patients who required more than 3 weeks to recover from any adverse effect.

\section{Evaluation of safety and efficacy}

Patients' data, including the results of imaging studies, were recorded in electronic clinical records. A multidisciplinary colon cancer team in the hospital confirmed patient eligibility. Adverse effects were graded according to Common Terminology Criteria for Adverse Events version 3.0 in all patients every 2 weeks or before each treatment cycle. Treatment was continued until disease progression, unmanageable toxic effects, patient refusal, or transfer of the patient to another hospital. Baseline tumor response was assessed within 2 weeks before enrollment in the study, and tumor response was then prospectively assessed every 6 weeks on computed tomography according to Response Evaluation Criteria for Solid Tumors version 1.0. Fluorine-18 fluorodeoxyglucose positive emission tomography/computed tomography was performed before and after NAC to identify further systemic metastases. In addition, all preoperative assessments were performed within 2 weeks before the scheduled day of surgery. An independent ethics committee confirmed all safety and efficacy data derived from this study.

\section{Surgical treatment}

Surgery was planned at least 3 weeks after the last dose of chemotherapy and 5 weeks after the last dose of bevacizumab.
Eligibility criteria for surgery were as follows: a preoperative tumor response of complete response, partial response, or stable disease; neutrophil count $\geq 1,500 / \mathrm{mm}^{3}$; platelet count $\geq 100,000 / \mathrm{mm}^{3}$; hemoglobin level $\geq 8.0 \mathrm{~g} / \mathrm{dL}$; total bilirubin $\leq 1.5$ times the ULN; aspartate aminotransferase, alanine aminotransferase, and alkaline phosphatase $\leq 2.5$ times the ULN; serum creatinine $\leq 1.5$ times the ULN; and R0 resection of all tumor sites expected.

\section{Statistical analysis}

The primary objective of this study was to assess the completion rate of NAC and the possibility of curative surgery. Secondary endpoints were the safety of perioperative chemotherapy, perioperative complications, progression-free survival (PFS), relapse-free survival (RFS), overall survival (OS), and the correlation between radiological tumor response and histological response. The overall response rate was based on the number of patients who had a complete or partial response to treatment. PFS was defined as the time between the date of starting treatment and the date of confirming disease progression or death. Data on patients without disease progression were censored on the date at which the patient was last confirmed to be alive. RFS was defined as the time between the date of operation and the date of confirming relapse or death; data on patients without relapse were censored at the time the patient was last confirmed to be alive. OS was calculated from the date of the first day of treatment until the date of death from any cause. In patients who were lost to follow-up, data were censored on the date the patient was last confirmed to be alive. PFS, RFS, and OS were estimated by the Kaplan-Meier method and compared using the log-rank test; predictive or prognostic factors were identified by univariate analysis.

All analyses were carried out using Statistical Package for the Social Sciences software version 22.0 (IBM Corporation, Armonk, NY, USA). $P<0.05$ was considered to indicate statistical significance. On the basis of previous clinical trials of FOLFOX, the expected treatment completion rate was assumed to be $60 \%$ given the advanced stage of CRC being treated. We estimated that 44 patients would be required for the study to achieve an expected treatment completion rate of $80 \%$, with a lower limit of $60 \%$, a one-sided $\alpha$-level of $0.05 \%$, and a power of $90 \%$. To account for exclusions from analysis, we decided that a total of 49 patients should be enrolled.

An interim analysis was planned after the 12th patient had received NAC and undergone surgery. If more than three of the first 12 patients completed the protocol treatment, 
including NAC and curative resection, the null hypothesis would be rejected according to the exact test based on a binomial distribution. Thus, this study was to be terminated if the number of patients completing the protocol treatment was three or less.

\section{Results}

\section{Study process and termination}

A total of 12 patients were enrolled between July 2007 and December 2011. The planned interim analysis was performed, and the criteria for continuation of the study were met. However, in December 2012, we decided to stop this Phase II study because of slow registration. We hereby present the results of the interim analysis as the final report of this study. The median follow-up time was 56 months.

\section{Patient baseline characteristics}

The characteristics of the patients $(n=12)$ were as follows: median age, 60.5 (range 37-71) years; Eastern Cooperative Oncology Group performance status 0, 100\%; and disease stage IIIb/IV, 67\%/33\%. The detailed characteristics are shown in Table 1 . All patients were eligible for safety and efficacy analyses of NAC. One patient did not receive surgery because of non-treatment-related adverse events and was transferred to another hospital. This patient was therefore excluded from the PFS and RFS analysis sets.

\section{Treatment}

All 12 patients received six cycles of FOLFOX4 plus bevacizumab (bevacizumab withheld in the sixth cycle). The completion rate of NAC was $100 \%$ at the interim analysis. The median cumulative doses of each agent were 510 (range 410-510) mg/m² for oxaliplatin, 12,000 (range $10,000-12,000) \mathrm{mg} / \mathrm{m}^{2}$ for bolus and infusional 5-fluorouracil, and $25 \mathrm{mg} / \mathrm{kg}$ for bevacizumab. The median relative dose intensities for each agent were 0.94 (range 0.54-1.01) for both oxaliplatin and 5-fluorouracil and 0.94 (range 0.67-1.01) for bevacizumab. Treatment was delayed in five patients $(41.7 \%)$ because of grade 3 or higher neutropenia, grade 3 diarrhea, and poor general condition. The doses of oxaliplatin and 5-fluorouracil were reduced in three of these patients.

\section{Efficacy}

Tumor response was assessed before surgical resection in 12 patients. The response rate was $75 \%$ (95\% confidence interval 46-104). No patient had a best overall response of complete response or progressive disease. The response rate was $75 \%$ and did not differ between stage III and stage IV disease. As for the relationship between pretreatment stage
Table I Baseline characteristics of patients $(n=12)$

\begin{tabular}{|c|c|}
\hline Characteristic & n (\%) \\
\hline Sex, male/female & $8(66.7) / 4(33.3)$ \\
\hline Median age (range), years & $60.5(37-7 I)$ \\
\hline \multicolumn{2}{|l|}{ ECOG performance status } \\
\hline 0 & $12(100)$ \\
\hline$<1$ & 0 \\
\hline \multicolumn{2}{|l|}{ Site of primary tumor } \\
\hline Colon & $3(25)$ \\
\hline Rectum & $9(75)$ \\
\hline \multicolumn{2}{|l|}{ Histology } \\
\hline Well differentiated & $5(4 \mid .7)$ \\
\hline Moderately differentiated & $5(41.7)$ \\
\hline Poorly differentiated & I (8.3) \\
\hline Mucinous & I (8.3) \\
\hline \multicolumn{2}{|l|}{ Clinical stage } \\
\hline III & $8(66.7)$ \\
\hline IV & $4(33.3)$ \\
\hline \multicolumn{2}{|l|}{ Clinical T stage } \\
\hline T3, invades through muscularis propria & $7(58.3)$ \\
\hline $\mathrm{T} 4$, penetrates to peritoneum & $5(41.7)$ \\
\hline \multicolumn{2}{|l|}{ Clinical $N$ stage } \\
\hline $\mathrm{NI}$ & I (8.3) \\
\hline N2 & II (9I.7) \\
\hline \multicolumn{2}{|l|}{ Sites of metastases } \\
\hline Liver & $3(25)$ \\
\hline PALN & $\mathrm{I}(8.3)$ \\
\hline \multicolumn{2}{|l|}{ KRAS gene status } \\
\hline Wild-type & $5(4 I .7)$ \\
\hline Mutant & $2(1.7)$ \\
\hline Unknown & $5(41.7)$ \\
\hline
\end{tabular}

Abbreviations: ECOG, Eastern Cooperative Oncology Group; PALN, para-aortic lymph nodes.

and pathological stage in the eleven patients who underwent surgery, one patient $(9.1 \%)$ had downstaging of T stage from T4 (tumor penetrating the visceral peritoneum) to T3. Seven patients $(63.6 \%)$ had downstaging of $\mathrm{N}$ stage: from $\mathrm{N} 2$ to N0 $(n=2)$ or $\mathrm{N} 1(\mathrm{n}=4)$ in six patients $(54.5 \%)$, and from N1 to N0 in one patient $(9.1 \%)$. There was no upstaging of $\mathrm{T}$ or $\mathrm{N}$ stage as compared with the pretreatment evaluation. Fluorine-18 fluorodeoxyglucose positive emission tomography/computed tomography was performed in all patients before and after NAC, and showed no evidence of tumor progression or new lesions. Radiologically, all patients thus showed some effect of NAC.

PFS, RFS, and OS were analyzed in the eleven patients who underwent surgery. Median PFS was 18.2 months $(95 \%$ confidence interval, not reached), RFS was 14.7 months $(95 \%$ confidence interval, not reached), and median OS was not reached (Figure 1). During follow-up, six patients relapsed but only one patient died of CRC. More than half of the patients survived over 5 years at the study cut-off point.

Univariate analysis was performed and PFS was significantly longer in stage IIIb disease than in stage IV disease 

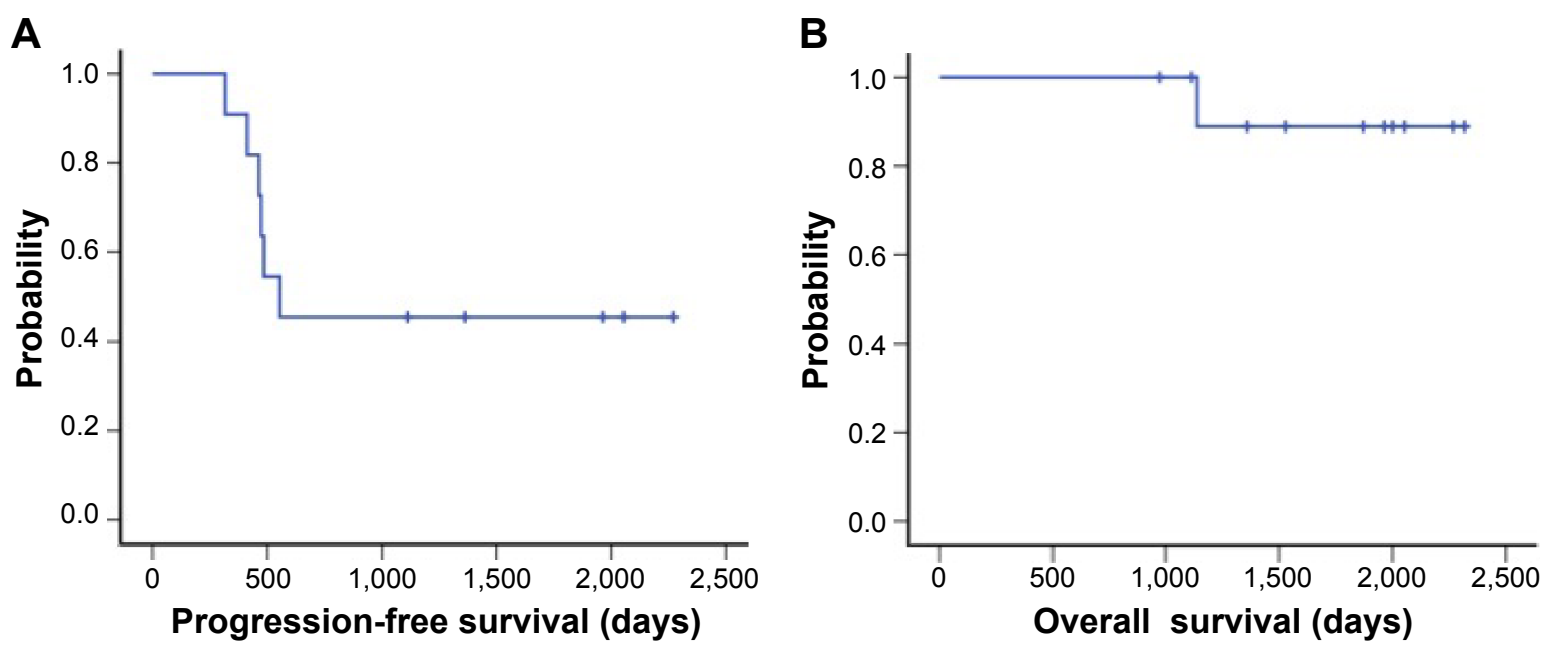

Figure I Progression-free survival (A) and overall survival (B) in patients with advanced colorectal cancer treated with FOLFOX4 plus bevacizumab.

(log-rank, $P=0.002$ ), and a similar trend was also seen in RFS (log-rank, $P=0.01)$. However, OS did not differ significantly between stage III and IV (log-rank, $P=0.157$, Figure 2$)$. In addition, OS was did not differ significantly between patients with relapse and those without relapse (log-rank, $P=0.371$ ).

\section{Safety}

Adverse events are summarized in Table 2. The most common grade 3 or 4 adverse events were neutropenia $(41.7 \%)$ and diarrhea (8.2\%). As for bevacizumab-related adverse events, no patient had severe bleeding, thromboembolic events, or gastrointestinal perforation. There were no other treatmentrelated severe adverse events or deaths during NAC.

\section{Surgery}

The median intervals from the last doses of bevacizumab and FOLFOX4 to surgery were 51 (range 41-63) days and
34 (range 27-41) days, respectively. Eleven patients (91.7\%) underwent curative surgery (R0), including resection of all metastatic lesions. Two patients had perioperative complications, ie, an anastomotic leak in one and wound infection in the other. These complications were reversible and resolved. There were no other severe complications or life-threatening events before or after surgery. Histological examination of resected specimens of the primary tumor revealed a minor response in nine patients and a major response in two patients. In addition, liver metastases showed a major response in two patients.

Adjuvant chemotherapy was received by eleven patients, but the start of treatment was delayed and deviated from the protocol in one patient who had an anastomotic leak. The median interval from the day of surgery to the start of adjuvant chemotherapy was 37 (range 28-42) days in the other patients. All patients received six cycles of FOLFOX4.
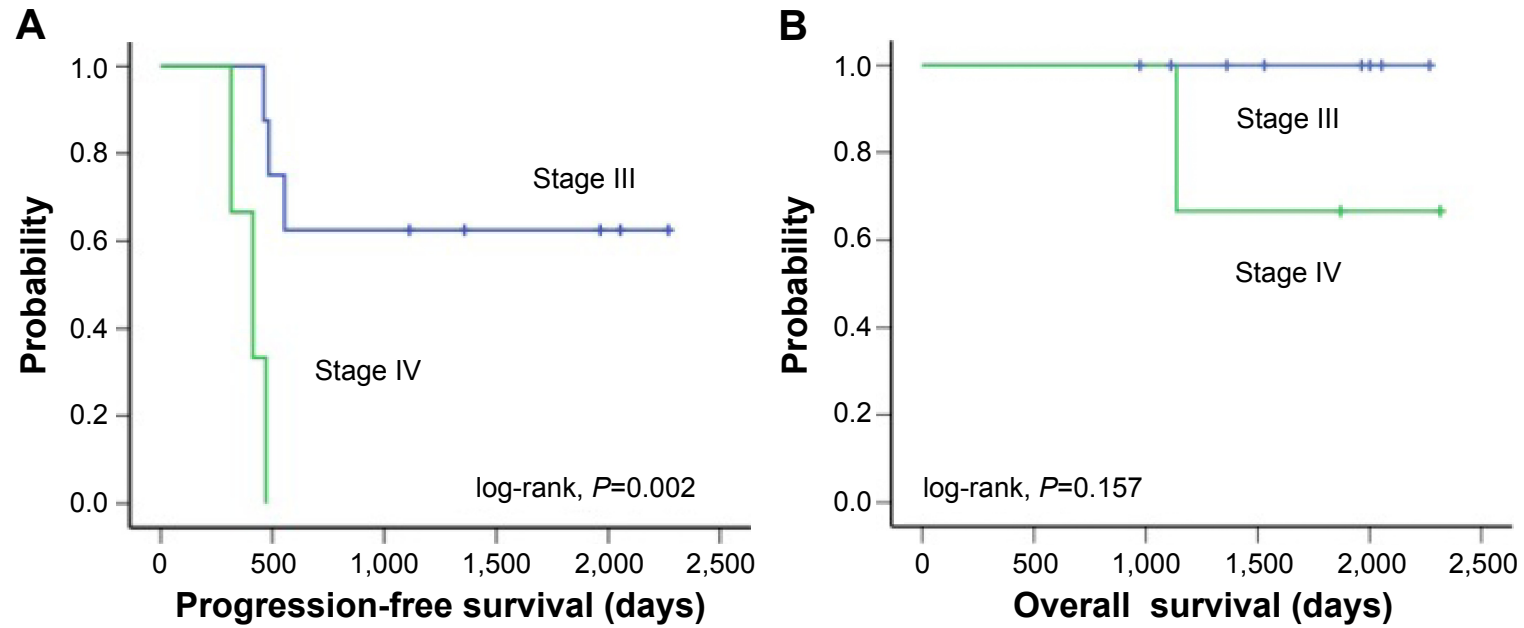

Figure 2 Progression-free survival $(\mathbf{A})$ and overall survival $(\mathbf{B})$ in patients with stage III (log-rank, $P=0.002)$ and stage IV (log-rank, $P=0$. I57) colorectal cancer treated with FOLFOX4 plus bevacizumab. 
Table 2 Adverse events according to Common Terminology Criteria for Adverse Events version $3.0(n=12)$

\begin{tabular}{|c|c|c|}
\hline \multirow[t]{2}{*}{ Adverse events } & \multirow{2}{*}{$\frac{\text { All grades }}{\mathrm{n}(\%)}$} & \multirow{2}{*}{$\begin{array}{l}\text { Grade } 3 \text { or higher } \\
\text { n (\%) }\end{array}$} \\
\hline & & \\
\hline \multicolumn{3}{|l|}{ Hematological } \\
\hline Leukopenia & $8(66.7)$ & $5(4 I .7)$ \\
\hline Neutropenia & $8(66.7)$ & $5(4 I .7)$ \\
\hline Anemia & $5(4 I .7)$ & 0 \\
\hline Thrombocytopenia & I (8.3) & 0 \\
\hline Febrile neutropenia & 0 & 0 \\
\hline \multicolumn{3}{|l|}{ Non-hematological } \\
\hline Anorexia & $8(66.7)$ & $8(66.7)$ \\
\hline Nausea & $3(25)$ & 0 \\
\hline Vomiting & I (8.3) & 0 \\
\hline Diarrhea & $4(33.3)$ & $\mathrm{I}(8.2)$ \\
\hline Stomatitis & $2(16.7)$ & 0 \\
\hline Hand-foot syndrome & $8(66.7)$ & 0 \\
\hline Total bilirubin increased & I (8.3) & 0 \\
\hline AST increased & $5(4 \mid .7)$ & 0 \\
\hline ALT increased & $3(25)$ & 0 \\
\hline Creatinine increased & I (8.2) & 0 \\
\hline Sensory neuropathy & $12(100)$ & 0 \\
\hline Allergic reaction & 0 & 0 \\
\hline
\end{tabular}

Abbreviations: AST, aspartate aminotransferase; ALT, alanine aminotransferase.

Two patients did not receive bevacizumab because of anastomotic leakage or wound infection. There was no severe adverse event or death during adjuvant chemotherapy.

\section{Treatment after relapse}

The outcomes of the enrolled patients are summarized in Table 3. Six of eleven patients followed up in our hospital had recurrence of CRC. Three patients received radical resection for locoregional or liver metastases, and the other three patients received systemic chemotherapy, including oxaliplatin, irinotecan, bevacizumab, and cetuximab. One of the latter three patients died.

\section{Discussion}

To our knowledge, this is the first Phase II study to evaluate the safety and efficacy of perioperative chemotherapy in patients with initially unresectable advanced CRC, including metastatic lesions. Although the results of interim analyses indicated that this strategy is highly active, the study was prematurely discontinued because of very slow patient enrollment. Because the protocol required that patients had advanced disease, the participating surgeons were concerned that preoperative chemotherapy might delay surgery and increase the risk of stenosis, obstruction, or bleeding caused by disease progression, thereby precluding radical surgery. Such uneasiness is often experienced by Japanese surgeons, although oxaliplatin-based adjuvant chemotherapy is now commonly used to treat patients with high-risk stage II or stage III CRC. Adjuvant chemotherapy including oxaliplatin without any molecularly targeted agents has been shown to improve RFS or OS; however, outcomes remain inadequate. ${ }^{11-13}$ Hence, the long-term aim of our study was to improve PFS and OS by perioperative chemotherapy and thereby improve the outcomes of patients with CRC as a whole. In fact, NAC has recently received considerable attention as the strategy most likely to further improve outcomes in patients with advanced CRC because the development of regimens for adjuvant chemotherapy has now reached a deadlock.

Table 3 Summary of patient outcomes $(n=12)$

\begin{tabular}{|c|c|c|c|c|c|c|c|c|c|c|}
\hline Case & Sex & $\begin{array}{l}\text { Age, } \\
\text { years }\end{array}$ & $\begin{array}{l}\text { Primary } \\
\text { site }\end{array}$ & $\begin{array}{l}\text { Metastatic } \\
\text { site }\end{array}$ & Stage & RECIST & $\begin{array}{l}\text { Resection } \\
\text { margin }\end{array}$ & $\begin{array}{l}\text { Recurrence } \\
\text { site }\end{array}$ & Post-relapse & Outcome \\
\hline I & $\mathrm{F}$ & 53 & $\mathrm{Ra}$ & PALN & IV & SD & Ro & $\begin{array}{l}\text { Peritoneum } \\
\text { (solitary) }\end{array}$ & Surgery & Still alive \\
\hline 2 & $M$ & 52 & $\mathrm{Ra}$ & Liver & IV & PR & $\mathrm{N} / \mathrm{A}$ & N/A & $\mathrm{N} / \mathrm{A}$ & $\mathrm{N} / \mathrm{A}$ \\
\hline 3 & $\mathrm{~F}$ & 67 & $\mathrm{Ra}$ & & III & PR & Ro & None & $\mathrm{N} / \mathrm{A}$ & Still alive \\
\hline 4 & $M$ & 65 & $\mathrm{Ra}$ & & III & PR & Ro & None & $\mathrm{N} / \mathrm{A}$ & Still alive \\
\hline 5 & $\mathrm{~F}$ & 58 & $S$ & Liver & IV & PR & Ro & LN & $\begin{array}{l}\text { FOLFIRI + BV, } \\
\text { CPT + CET }\end{array}$ & Dead \\
\hline 6 & $M$ & 50 & $\mathrm{Ra}$ & & III & SD & Ro & Local & Surgery & Still alive \\
\hline 7 & $\mathrm{~F}$ & 71 & A & & III & SD & Ro & None & $\mathrm{N} / \mathrm{A}$ & Still alive \\
\hline 8 & M & 40 & Rs & Liver & IV & PR & Ro & Liver & Surgery & Still alive \\
\hline 9 & $M$ & 66 & $\mathrm{Ra}$ & & III & PR & Ro & Lung & $\begin{array}{l}\text { XELOX + BV, } \\
\text { FOLFIRI + BV }\end{array}$ & Still alive \\
\hline 10 & $M$ & 63 & $\mathrm{Ra}$ & & III & PR & Ro & None & $\mathrm{N} / \mathrm{A}$ & Still alive \\
\hline II & $M$ & 37 & $\mathrm{Ra}$ & & III & PR & Ro & None & $\mathrm{N} / \mathrm{A}$ & Still alive \\
\hline 12 & M & 67 & $S$ & & III & PR & Ro & Peritoneum & XELOX & Still alive \\
\hline
\end{tabular}

Abbreviations: Ra, rectum/above the peritoneal reflection; Rs, rectosigmoid; S, sigmoid colon; A, ascending colon; PALN, para-aortic lymph nodes; SD, stable disease; PR, partial response; R0, complete resection; BV, bevacizumab; N/A, not applicable; RECIST, Response Evaluation Criteria for Solid Tumors. 
The Phase III FOxTROT trial was the first to evaluate the effectiveness of preoperative chemotherapy in locally advanced colon cancer. ${ }^{14}$ In the pilot stage, 150 patients with radiologically confirmed $\mathrm{T} 3$ ( $\geq 5 \mathrm{~mm}$ invasion beyond the muscularis propria) or T4 tumors were randomly assigned in a 2:1 ratio to receive preoperative chemotherapy with three cycles ( 6 weeks) of oxaliplatin plus modified de Gramont infusional fluorouracil (OxMdG) followed by surgery and another nine cycles (18 weeks) of OxMdG or to receive standard postoperative chemotherapy with 12 cycles ( 24 weeks) of OxMdG. In addition, patients with $K R A S$ wild-type tumors in each arm were randomly assigned to receive panitumumab during the first 6 weeks of chemotherapy or not. The objective of the study was to assess whether preoperative treatment with 6 weeks of the standard UK OxMdG regimen with or without panitumumab improves disease-free survival in patients with locally advanced, potentially resectable colon cancer. Whether tumor shrinkage was enhanced by adding panitumumab was also assessed as compared with chemotherapy alone.

The first report of the FOxTROT study documented that preoperative chemotherapy is feasible with a good tumor response. Data on RFS and OS have yet to be reported. The results of the FOxTROT study indicated that preoperative chemotherapy was associated with significant tumor downstaging as well as significantly less apical node involvement and resection margin or peritoneal margin involvement as compared with postoperative chemotherapy. There were no significant differences in the rates of perioperative complications or toxicity due to chemotherapy between the two groups, and the chemotherapy completion rate among patients who received postoperative chemotherapy was higher in the preoperative group than in the postoperative group $(82 \%$ versus $73 \%$ ). Incipient obstruction did not develop in any patient during preoperative chemotherapy. However, one patient in each group required acute surgery because of incipient obstruction, but proceeded to curative surgery. The pilot phase of the FOxTROT trial thus concluded that preoperative chemotherapy for locally advanced operable primary colon cancer is feasible, with acceptable toxicity and perioperative morbidity, and recommended to proceed to the Phase III trial to investigate long-term oncological outcomes. Thus, new evidence supporting the effectiveness of preoperative chemotherapy for locally advanced operable colon cancer is expected to change the treatment algorithm in high-risk stage II or III colon cancer in the near future.

Because one of our objectives was to define the characteristics of patients with stage IV CRC most likely to benefit from NAC, we studied not only patients with hepatic metastases but also those with simultaneous extrahepatic metastases. Several studies have evaluated perioperative chemotherapy in patients with $\mathrm{CRC}$ and metastases limited to the liver, but not in those with extrahepatic metastases. The EORTC40983 trial (a Phase III EPOC trial) compared a perioperative chemotherapy group (six cycles of FOLFOX4 before and after surgery) with a surgery alone group in patients with initially resectable liver metastases. A significant benefit in terms of PFS was obtained only in eligible or resected patients, but not randomly assigned patients. ${ }^{15}$ On long-term follow-up to assess OS, perioperative chemotherapy has shown no survival benefit.

Another Phase III study, the New EPOC trial, was performed to investigate whether the addition of cetuximab to perioperative chemotherapy was more beneficial than perioperative chemotherapy alone in terms of PFS in patients with $K R A S$ wild-type tumors who had initially resectable liver metastases. ${ }^{16}$ However, cetuximab was apparently not beneficial. On the contrary, addition of cetuximab to chemotherapy negatively affected PFS as well as OS. The New EPOC trial concluded that combining cetuximab with chemotherapy in a perioperative setting cannot be recommended. Thus, perioperative chemotherapy with FOLFOX has become a widely used, albeit marginally effective, treatment strategy.

Two pivotal, non-randomized Phase II studies have evaluated bevacizumab plus chemotherapy as perioperative treatment. ${ }^{17,18}$ Gruenberger et al assessed the efficacy and safety of six cycles of bevacizumab plus capecitabine and oxaliplatin before surgery in patients with potentially resectable high-risk CRC with liver metastases. ${ }^{17} \mathrm{~A}$ high objective response rate of $73 \%$ was obtained, with no increase in intraoperative bleeding, wound healing complications, or postoperative mortality. The authors suggested that bevacizumab can be administered safely as perioperative treatment for liver metastases without increasing the risk of severe complications. In the other study, the safety and efficacy of perioperative treatment with capecitabine, oxaliplatin, and bevacizumab were confirmed in patients with initially unresectable CRC liver metastases. ${ }^{18}$ On the basis of these results, bevacizumab plus chemotherapy has been considered a candidate standard strategy for the perioperative treatment of CRC liver metastases, thus supporting our study concept.

In our study, PFS and RFS were significantly better in stage III disease than in stage IV disease; there was no difference in OS between these two subgroups. Interestingly, median OS was longer than 5 years, regardless of PFS events. 
In most previous Phase III trials of standard chemotherapy plus biological agents, the median OS was between 2 and 3 years in patients with metastatic CRC, but our results considerably exceeded this goal in survival. Our findings suggest that FOLFOX4 plus bevacizumab is likely to improve survival in patients who have advanced CRC with or without potentially resectable metastatic lesions, associated with a high risk of tumor relapse despite perioperative chemotherapy combined with bevacizumab.

In conclusion, although this was an interim analysis of outcomes in a small study group, our results suggest that perioperative chemotherapy with FOLFOX4 plus bevacizumab is feasible and has a high response rate, leading to curative surgery and prolonging OS in patients with initially unresectable advanced $\mathrm{CRC}$, including those who concurrently have potentially resectable metastatic lesions.

\section{Acknowledgment}

We thank the participating patients, their family members, and all researchers involved in this research. We are grateful to the staff of the ambulatory treatment center for their management of the patients recruited for this study. The results of this study were presented in abstract form at the 2012 American Society of Clinical Oncology Annual Meeting.

\section{Disclosure}

The authors have no conflicts of interest in this work.

\section{References}

1. National Cancer Center, Center for Cancer Control and Information Services. Cancer Statistics in Japan, 2012. Available from: http://ganjoho.jp/ public/statistics/backnumber/2012_en.html. Accessed April 19, 2015.

2. Yoshino T, Mizunuma N, Yamazaki K, et al. TAS-102 monotherapy for pretreated metastatic colorectal cancer: a double-blind, randomised, placebo-controlled Phase 2 trial. Lancet Oncol. 2012;13:993-1001.

3. Hurwitz H, Fehrenbacher L, Novotny W, et al. Bevacizumab plus irinotecan, fluorouracil and leucovorin for metastatic colorectal cancer. N Engl J Med. 2004;350:2335-2342.

4. Kabbinavar F, Hurwitz HI, Fehrenbacher L, et al. Phase II, randomized trial comparing bevacizumab plus fluorouracil (FU)/leucovorin (LV) with FU/LV alone in patients with metastatic colorectal cancer. $J$ Clin Oncol. 2003;21:60-65.

OncoTargets and Therapy

\section{Publish your work in this journal}

OncoTargets and Therapy is an international, peer-reviewed, open access journal focusing on the pathological basis of all cancers, potential targets for therapy and treatment protocols employed to improve the management of cancer patients. The journal also focuses on the impact of management programs and new therapeutic agents and protocols on

Submit your manuscript here: http://www.dovepress.com/oncotargets-and-therapy-journal
5. Kabbinavar FF, Schulz J, McCleod M, et al. Addition of bevacizumab to bolus fluorouracil and leucovorin in first-line metastatic colorectal cancer: results of a randomized Phase II trial. J Clin Oncol. 2005;23:3697-3705.

6. Saltz LB, Clarke S, Díaz-Rubio E, et al. Bevacizumab in combination with oxaliplatin-based chemotherapy as first-line therapy in metastatic colorectal cancer: a randomized Phase III study. J Clin Oncol. 2007;26:2013-2019.

7. Giantonio BJ, Catalano PJ, Meropol NJ, et al. Bevacizumab in combination with oxaliplatin, fluorouracil, and leucovorin (FOLFOX4) for previously treated metastatic colorectal cancer: results from the Eastern Cooperative Oncology Group Study E3200. J Clin Oncol. 2006;25:1539-1544.

8. Robinson S, Manas DM, Pedley I, et al. Systemic chemotherapy and its implications for resection of colorectal liver metastasis. Surg Oncol. 2011;20:57-72.

9. Nordlinger B, Van Cutsem E, Gruenberger T, et al. Combination of surgery and chemotherapy and the role of targeted agents in the treatment of patients with colorectal liver metastases: recommendations from an expert panel. Ann Oncol. 2009;20:985-992.

10. Japanese Society for Cancer of the Colon and Rectum. General rules for clinical and pathological studies on cancer of the colon, rectum and anus. Part I. Clinical classification. Surg Today. 1983;13:557-573.

11. André T, Boni C, Mounedji-Boudiaf L, et al. Oxaliplatin, fluorouracil, and leucovorin as adjuvant treatment for colon cancer. $N$ Engl J Med. 2004;350:2343-2351.

12. Kuebler JP, Wieand HS, O'Connell MJ, et al. Oxaliplatin combined with weekly bolus fluorouracil and leucovorin as surgical adjuvant chemotherapy for stage II and III colon cancer: results from NSABP C-07. J Clin Oncol. 2007;25:2198-2204.

13. de Gramont A, Van Cutsem E, Schmoll HJ, et al. Bevacizumab plus oxaliplatin-based chemotherapy as adjuvant treatment for colon cancer (AVANT): a Phase 3 randomised controlled trial. Lancet Oncol. 2012;13:1225-1233.

14. FoxTROT Collaborative Group. Feasibility of preoperative chemotherapy for locally advanced, operable colon cancer: the pilot phase of a randomised controlled trial. Lancet Oncol. 2012;13:1152-1160.

15. Nordlinger B, Sorbye H, Glimelius B, et al. Perioperative chemotherapy with FOLFOX4 and surgery versus surgery alone for resectable liver metastases from colorectal cancer (EORTC Intergroup trial 40983): a randomised controlled trial. Lancet. 2008;371:1007-1016.

16. Primrose J, Falk S, Finch-Jones M, et al. Systemic chemotherapy with or without cetuximab in patients with resectable colorectal liver metastasis: the New EPOC randomised controlled trial. Lancet Oncol. 2014;15:601-611.

17. Gruenberger B, Tamandl D, Schueller J, et al. Bevacizumab, capecitabine, and oxaliplatin as neoadjuvant therapy for patients with potentially curable metastatic colorectal cancer. J Clin Oncol. 2008; 26:1830-1835.

18. Wong R, Cunningham D, Barbachano Y, et al. A multicentre study of capecitabine, oxaliplatin plus bevacizumab as perioperative treatment of patients with poor-risk colorectal liver-only metastases not selected for upfront resection. Ann Oncol. 2011;22:2042-2048. patient perspectives such as quality of life, adherence and satisfaction. The manuscript management system is completely online and includes a very quick and fair peer-review system, which is all easy to use. Visit http://www.dovepress.com/testimonials.php to read real quotes from published authors. 